\title{
Modeling Foamy Oil Flow in Porous Media II: Nonlinear Relaxation Time Model of Nucleation
}

\author{
D.D. Joseph, A.M Kamp ${ }^{\dagger}$, T. Ko, R. Bai \\ University of Minnesota \\ ${ }^{\dagger}$ Intevep SA, PDVSA, Venezuela
}

May 2002

\begin{abstract}
In a previous communication, here called Part I, we presented a model of the flow of foamy oil in porous media in situations in which the bubbles do not coalesce to produce the percolation of free gas so that the gas moves with the oil as it evolves. A central role in that theory is an equation of state, called the solubility isotherm, which describes an equilibrium between the fraction of dispersed gas $\phi$ and the pressure below the bubble point pressure. A rate equation governing the return to equilibrium was postulated and it requires a value for the relaxation constant multiplying $\phi$. The theory developed in Part I was applied to experimental data and good agreements were achieved except for sharp transients at early times such as those that occur for sudden drops of pressure at the open end of the closed sand pack. In the present theory we introduce two rates and two relaxation times to describe the dynamics of relaxation of the system to an equilibrium state; one rate for $\phi$ and the other for the pressure $p$ as was suggested already in Part I. However, we found that constant relaxation times could not be found to fit all the available data. We interpret this in terms of bubbles nucleating more slowly at initial drawdowns, and more rapidly as gas and vapor is released when the pressure is held below the bubble point. This feature has been more or less successfully addressed by the introduction of two relaxation functions of the gas fraction $\phi$ which allows us to describe the low rates of evolution of $\phi$ when $\phi$ is zero or close to it. The relaxation functions were fit to one rate of depletion in a depletion experiment in which oil is pulled out of a closed sand pack at a constant rate. With this selection of the relaxation function established, the set of governing PDEs is fixed and may be used to predict the results of other experiments. The prediction of the pressure profiles for other greatly different rates of depletion is satisfactory. Moreover, the experimental results processed in Part I, are improved by the new theory with the same fitting.
\end{abstract}

\section{Introduction}

The theory presented here can be advertised as a continuum theory for the nucleation and motion of dispersed gas in a porous media under the assumption that the bubbles move with the oil. The main novel features of theory are an equilibrium equation of state relating the gas fraction to the pressure, which was derived in Joseph, Kamp and Bai (2002), hereafter called Part I, and the nonlinear relaxation function that describes bubble nucleation and growth.

The motivation for this work arises from the anomalous high recovery factor and rates of production from reservoirs of heavy foamy oil under solution gas drive. All of the background motivation, the arguments justifying the model and detailed derivations were presented in Part I and will not be repeated here. This paper can be viewed as the next chapter in the derivation of a continuum theory of flow of foamy oil in porous media and we did not prepare it to stand alone. 


\section{Governing equations}

First we shall lay down the governing equations used in this work and tell readers how they reduce to equations used in Part I, calling attention to some important features. The equations to be solved are as follows.

$$
\begin{gathered}
\mathbf{u}=-\lambda \nabla p \\
\frac{D \phi}{D t}-(1-\phi) \operatorname{div} \mathbf{u}=0 \\
\tau_{1} \frac{D}{D t}\left(\frac{\phi}{1-\phi}\right)+\tau_{2} \frac{D}{D t}\left(\frac{\tilde{p}-p}{p}\right)=\frac{\tilde{p}-p}{p}-\beta \frac{\phi}{1-\phi}
\end{gathered}
$$

where

$$
\frac{D(\bullet)}{D t} \stackrel{\text { def }}{=} \alpha \frac{\partial(\bullet)}{\partial t}+\mathbf{u} \cdot \nabla(\bullet)
$$

is a material derivative following the motion with mixture velocity $\mathbf{u}$ in a porous media of porosity $\alpha$. The gas fraction is $\phi, 0 \leq \phi \leq 1$ and $1-\phi$ is the liquid fraction. The pressure $p \leq \widetilde{p}$, where $\tilde{p}$ is the bubble point pressure; $\phi=0$ when $p=\tilde{p} . \tau_{1}(\phi)$ and $\tau_{2}(\phi)$ are relaxation functions chosen to fit data. $\beta$ is a solubility parameter that represents the solubility of reservoir gases (mainly methane) in the heavy oil (see table 1).

Equation (2.1) is Darcy's law and

$$
\lambda=\frac{k}{\eta(\phi)}
$$

is the mobility, $k$ is the permeability and $\eta(\phi)$ is the mixture viscosity. In Part I, we presented arguments for a viscosity function of the form

$$
\eta(\phi)=\frac{\eta(0)}{(1-\phi)^{m}}
$$

The expression (2.6) is supposed to describe the viscosity of the dispersion, even when the bubbles pack and the gas dispersion is a foam, with $\phi$ near to one giving the exceedingly high viscosity of a dry foam. $\eta(0)$ is the viscosity of the dry oil at the bubble point and $m$ is a fitting parameter. Note that (2.6) includes the viscosity increase at increasing $\phi$ both due to the generation of a dispersion and due to composition change (outgassing) of the continuous phase.

The equation (2.2) is the continuity equation for a mixture of incompressible fluids in which the density of the mixture is

$$
\rho(\phi)=\rho_{l}(1-\phi)+\rho_{G} \phi \approx \rho_{l}(1-\phi)
$$

because $\rho_{G}<<\rho_{l}$ with $\rho_{G}$ the gas density and $\rho_{l}$ the liquid density. It is assumed that the live oil is incompressible, though in fact oils of interest are slightly compressible. The theory does recognize the dissolved gas fraction in the oil (see Part I). 
Equation (2.3) is a constitutive equation governing the relaxation to equilibrium. The equilibrium is given by the solubility isotherm (derived in Part I)

$$
\begin{gathered}
\frac{\tilde{p}-p}{p}=\frac{\beta \phi}{1-\phi}, \\
\beta=\frac{T_{r e f}}{T} \frac{\hat{\gamma}}{p_{\text {ref }}}
\end{gathered}
$$

where $T$ is the absolute temperature and the reference is at a low $p$ is in which nearly all the gas has been released and $\hat{\gamma}$ is a constant which characterizes the change of pressure required to release gas of a certain volume in a PVT cell. (The implicit assumption here is that experiments in a PVT cell can be used to model bubble evolution in a porous medium occurring under thermodynamic equilibrium.) $\beta$ is a solubility coefficient, computed for some heavy oils in Part I and given in table 2.1 of Part I and reproduced in table 1 below.

\begin{tabular}{|r|c|c|}
\hline Oil & $\beta$ & $\mathbf{T}\left({ }^{\circ} \mathbf{K}\right)$ \\
\hline Lloydminster & 3.40 & 293 \\
\hline Lindbergh & 3.17 & 293 \\
\hline Cerro Negro & 3.53 & 327 \\
\hline
\end{tabular}

Table 1. Solubility coefficients for some heavy oils.

\section{Dimensionless equations and parameters}

Without losing generality, we may scale our equations in the one-dimensional case used to process sand pack experiments. After introducing dimensionless length $L$ and time $T$,

$$
X=x / L, \quad T=t / \theta
$$

and new dimensionless variables

$$
\left.\begin{array}{rl}
\Psi & =\frac{\phi}{(1-\phi)}, \\
P & =\frac{(\widetilde{p}-p)}{p}, \\
U & =\frac{u L}{\lambda_{0} \tilde{p}}, \\
\Lambda(\phi) & =\frac{\lambda(\phi)}{\lambda_{0}}=\left(\frac{1}{1+\Psi}\right)^{m}
\end{array}\right\},
$$

we get

$$
\begin{gathered}
U=\frac{\Lambda}{(P+1)^{2}} \frac{\partial P}{\partial X}=\frac{(1+\Psi)^{-m}}{(P+1)^{2}} \frac{\partial P}{\partial X}, \\
\frac{1}{(1+\Psi)}\left\{\frac{\partial \Psi}{\partial T}+U \frac{\partial \Psi}{\partial X}\right\}=\frac{\partial U}{\partial X},
\end{gathered}
$$




$$
J_{1}\left\{\frac{\partial \Psi}{\partial T}+U \frac{\partial \Psi}{\partial X}\right\}+J_{2}\left\{\frac{\partial P}{\partial T}+U \frac{\partial P}{\partial X}\right\}=P-\beta \Psi
$$

where

$$
\theta=\frac{\alpha L^{2}}{\lambda_{0} \widetilde{p}}
$$

is a characteristic time and

$$
J_{1}=\frac{\tau_{1} \tilde{p} \lambda_{0}}{L^{2}}, \quad J_{2}=\frac{\tau_{2} \tilde{p} \lambda_{0}}{L^{2}}
$$

are dimensionless relaxation times.

The equations (3.3), (3.4), and (3.5) are closed with selection of $m, J_{1}$ and $J_{2} ; \beta$ is known from table 1 .

In Part I we considered the simpler fitting problem in which $J_{2}=0$. The best values

$$
J_{1}=5, \quad m=11, \quad \beta=3.4
$$

were determined by fitting Maini and Sarma's (1994) experiment for steady flow through an open sand pack. The predictions of transients in a drawdown experiment using (3.8) were not good.

\section{Boundary conditions}

We consider three different types of experiments: depletion, drawdown, and blowdown.

Kumar, Pooladi-Darvish and Okazawa (2000) carried out depletion experiments in which live oil is pulled at a constant rate out of a sand pack closed at one end. At the closed end

$$
U=0 \text {. }
$$

At the open end,

$$
U=c
$$

is prescribed. It is easy to eliminate $U$ from (3.4) and (3.5) using (3.3). This leads to a coupled pair of nonlinear PDE's for $P$ and $\Psi$. To solve these equations it is sufficient to prescribe a pressure boundary condition at the open and closed end. This corresponds to the physics of nucleation in which the evolution of $\Psi$ (or $\phi$ ) is controlled by the pressure.

In figure 6.1 we have reproduced a graph of the average pressure vs. time for different constant rates of depletion. The volumetric average pressure is calculated using the pressure obtained at 7 points along the length of the sand pack.

Before depletion the sand pack was pressurized at a value of $620 \mathrm{psi}$; this is overpressurization of $45 \mathrm{psi}$ above the bubble point pressure. Over-pressurization drives more gas into solution, and gas pockets more deeply into crevices, which are nucleation sites on the solid surfaces of the porous media. The over-pressurization probably has the effect of delaying the outgassing of dissolved gas as the pressure is dropped below the bubble point. The decline of the pressure with volume when the pressure is higher than the bubble point pressure is due to the compressibility of live oil which is ignored in our theory. The main striking feature of the 
experiments is the undershoot whose magnitude increases strongly with the rate of depletion; deep undershoots follow from rapid depletion. The undershoots are due to the fact that the drawdown is too fast to be followed by nucleation and transfer of dissolved gas to free gas, which takes its own (relaxation) time. Once the nucleation starts, the pressure rises again.

In a drawdown experiment, which was carried out by Maini and Sarma (1994), the pressure at the open end is dropped in a prescribed manner; (4.3) holds and

$$
P=c_{3}(\tau) \text { (prescribed at open end). }
$$

In a blowdown experiment the pressure is dropped suddenly to a constant value $c_{3}<\widetilde{P}$.

\section{Selection of model parameters}

Our goal was to predict the undershoot and at the same time not deteriorate the agreements already achieved in Part I with the experiments of Maini and Sarma (1994). We first tried to achieve this goal with the selection of two constant times of relaxation ${ }^{*}$. The best parameters are close to the values

$$
J_{1}=200, \quad J_{2}=-18, \quad m=9, \quad \beta=3.4 .
$$

These choices led to modest improvements over the agreement already obtained in Part I, but the undershoot in the depletion and the transients in the blowdown experiments were not obtained.

Having convinced ourselves that the transients would never be modeled by a relaxation theory with constant relaxation we implemented the idea that the lag in the time of nucleation could be modeled by a nonlinear relaxation function of $\phi$ which was very large when $\phi$ is near to zero and then drops to modest values as $\phi$ rises away from zero. A large coefficient of $D \phi / D t$ tends to suppress $D \phi / D t$ in the evolution of $\phi$ so that $D \phi / D t$ would be small when $\phi$ was. With such a nonlinear relaxation for $\phi$, a similar, less muted, evolution of $P$ would be expected. The following expressions were processed against depletion data of Kumar et al (2000) for $0.37 \mathrm{cc} / \mathrm{hr}$ and selected experimental data of Maini and Sarma (1994):

$$
J_{1}=a_{1} \exp \left(-b_{1} \phi\right)+c_{1}, J_{2}=a_{2} \exp \left(-b_{2} \phi\right)+c_{2}
$$

We found that values

$$
\left\{\begin{array}{l}
a_{1}=200 \\
b_{1}=500 \\
c_{1}=5
\end{array}\right\},\left\{\begin{array}{l}
a_{2}=-1.99 \\
b_{2}=200 \\
c_{2}=-0.01
\end{array}\right\},\left\{\begin{array}{l}
m=9 \\
\beta=3.4
\end{array}\right\}
$$

gave rise to the comparisons examined in section 6.

It is almost certain that somewhat better values than those given by (5.3) could be obtained but delicate processing for this set of experiments is not compatible with our perception that the present theory gives the trends, and even the values, "more or less."

We call to the reader's attention now and again later, that one set of coefficients (5.3) describe all the experiments, even of different authors, reviewed below.

\footnotetext{
${ }^{*}$ The numerical code used in Part I is due to R. Bai. The code used in the more difficult problem here was devised by T. Ko and is described in Appendix A.
} 


\section{Comparison with experiments}

Here we compare the predictions of the theory given by (3.3), (3.4), (3.5), (5.2), (5.3) subject to the boundary condition in section 4 which is appropriate to the experiments. To enhance understanding we will also present the comparisons with the constant relaxation time theory (5.1) in certain cases; no comparison is given in the undershoot case arising in the depletion experiments because undershoots do not arise in the constant relaxation theory.

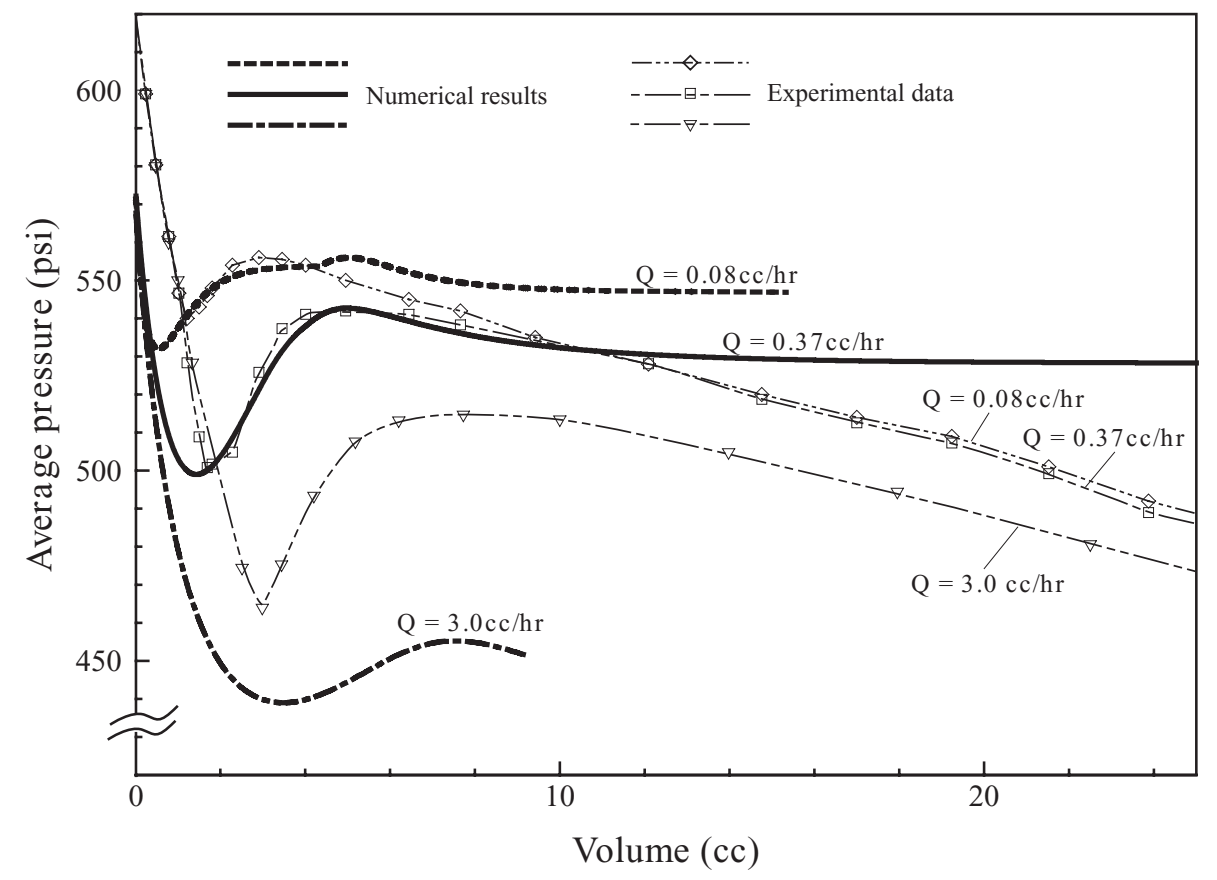

Figure 6.1. Comparison of the experimental data by Kumar, Pooladi-Darvish and Okazawa, (2000) and the numerical result with nonlinear relaxation at various depletion rate $Q=0.08,0.37$ and $3.0 \mathrm{cc} / \mathrm{hr}$ $\left(J_{1}=200 * \exp (-500 * \phi)+5, J_{2}=-1.99 * \exp (-200 * \phi)-0.01\right)$.

In figure 6.1, we compared the nonlinear relaxation theory with data from the depletion experiments of Kumar et al 2000. There is a disagreement for small depletion volumes and large depletion volumes. To understand the axes, note that with a constant rate of expansion

$$
\text { volume }(\mathrm{cc})=\dot{v} t
$$

where $\dot{v}=\mathrm{cc} / \mathrm{hr}$ and $t$ is in hours. The experiments have a finite slope at small times when the average pressure is above the saturation pressure $575 \mathrm{psi}$. The finite slope here tells how the volume of live oil, without bubbles, increases as pressure drops. This represents the compressibility of live oil; the live oil is assumed to be incompressible in the theory.

At large volumes (or times) the experimental pressure falls well below the predicted values. We believe that this is due to the coalescence and then percolation of free gas in the experiments which is ignored in the theory. 


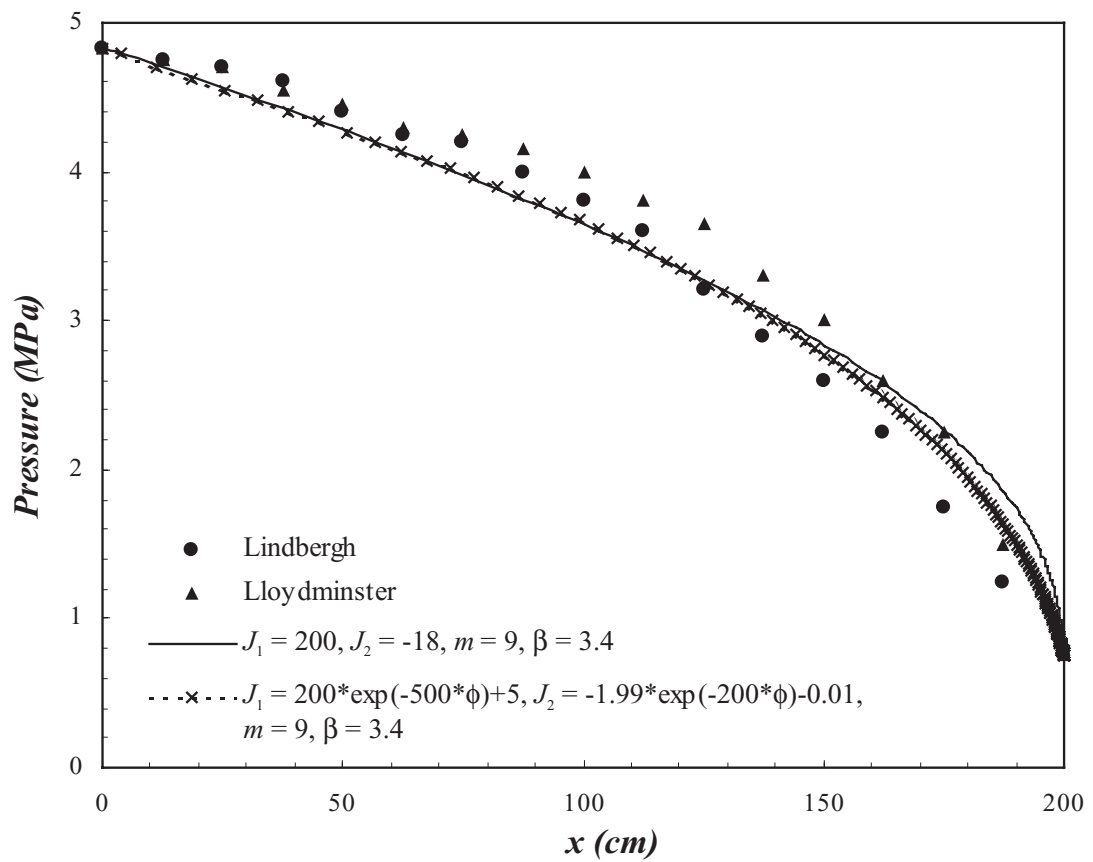

Figure 6.2. The pressure distribution for this steady problem at outlet pressure $P_{L}=0.75 \mathrm{MPa}$.

We turn next to a comparison of theory to the steady flow experiments of Maini and Sarma (1994). In figure 6.2 we compare the pressure distributions for the most unfavorable case of steady flow from a live oil reservoir to the outlet at a lower pressure. The nonlinear relaxation is better than the constant relaxation theory which is better than the single constant relaxation time given in Part I. In the other cases, in which the outlet pressure $P_{L}>0.75 \mathrm{MPa}$ the agreements are better and order in the same way as here.

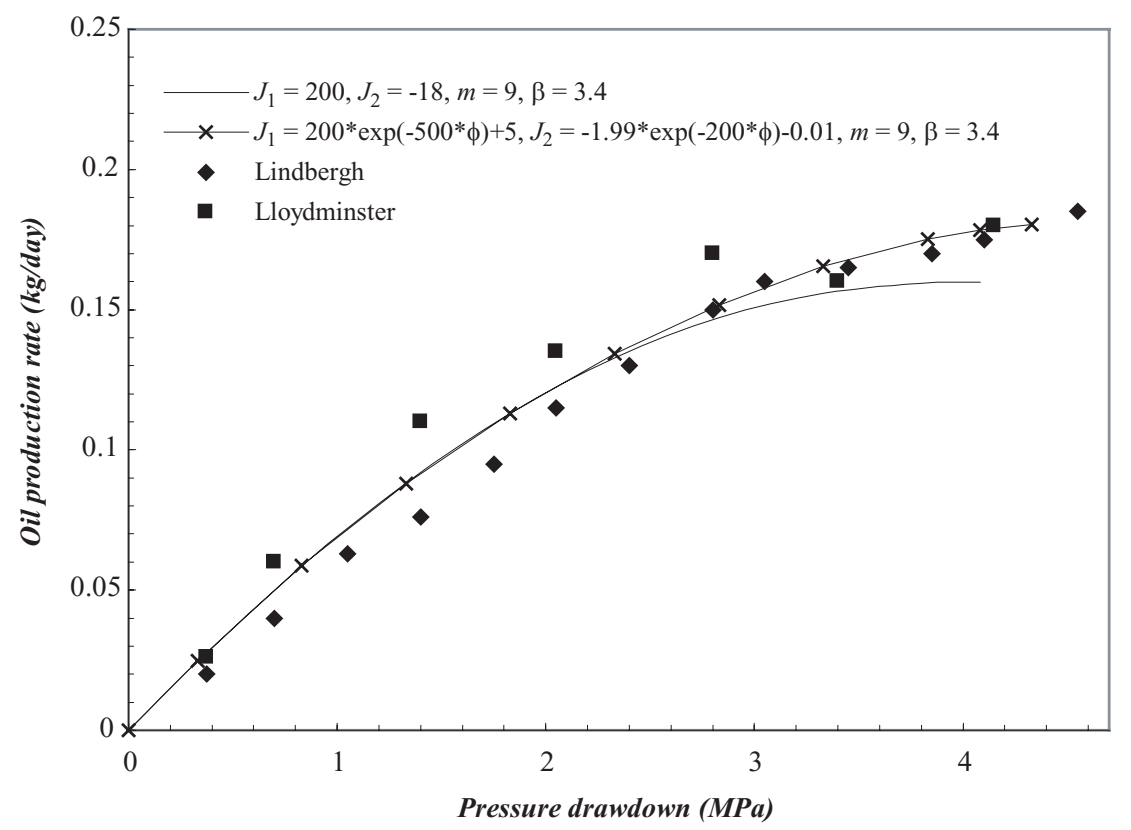

Figure 6.3. The oil production rate for steady problems as a function of the drawdown pressure. 
In figure 6.3 we plotted the oil production rate against the outlet pressure drawdown, which is the difference between the inlet and outlet pressure. The agreement between the nonlinear relaxation theory and the experiments is good.

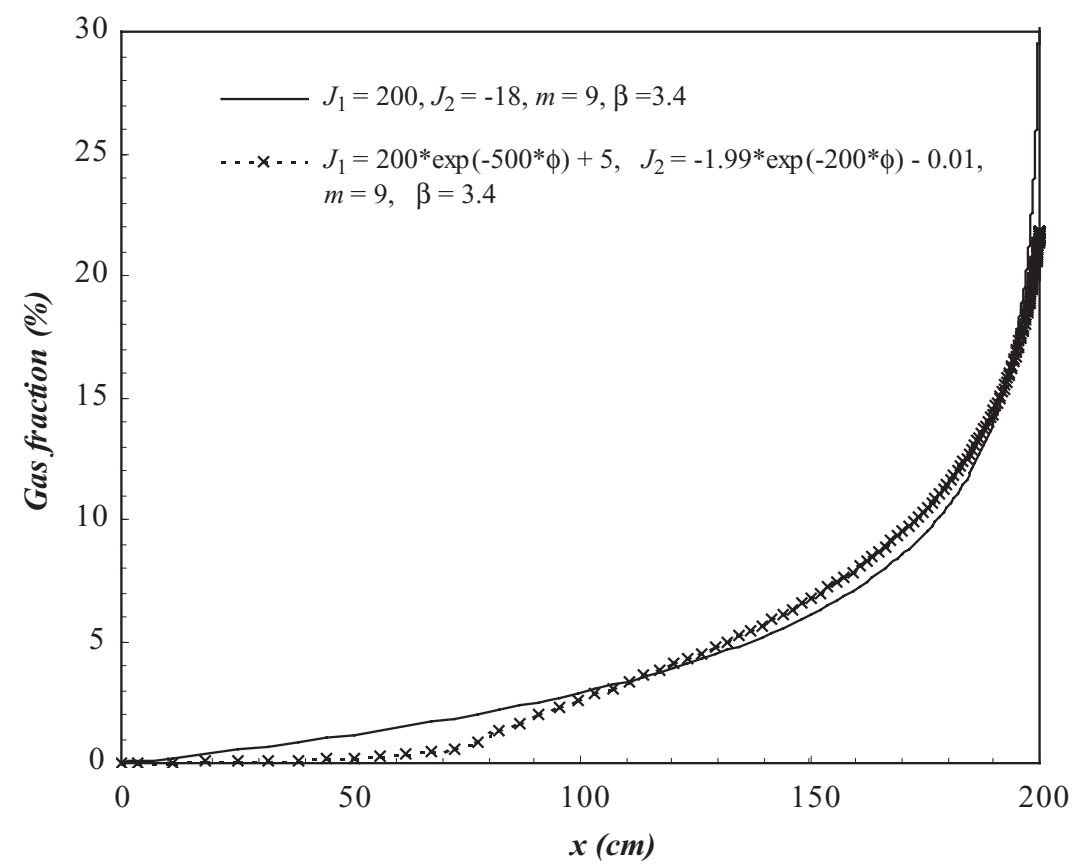

Figure 6.4. The gas fraction distribution for the steady problem at outlet pressure $P_{L}=0.75 \mathrm{MPa}$ (see figure 6.2).

In figure 6.4 we plotted the gas fraction distribution, $\phi$ vs. $x$ when the outlet pressure is lowest. There is no experimental data for this case. The figure shows that the nonlinear relaxation theory suppresses evolution of gas when the gas fraction is small, consistent with our understanding of nucleation, and growth of bubbles. 


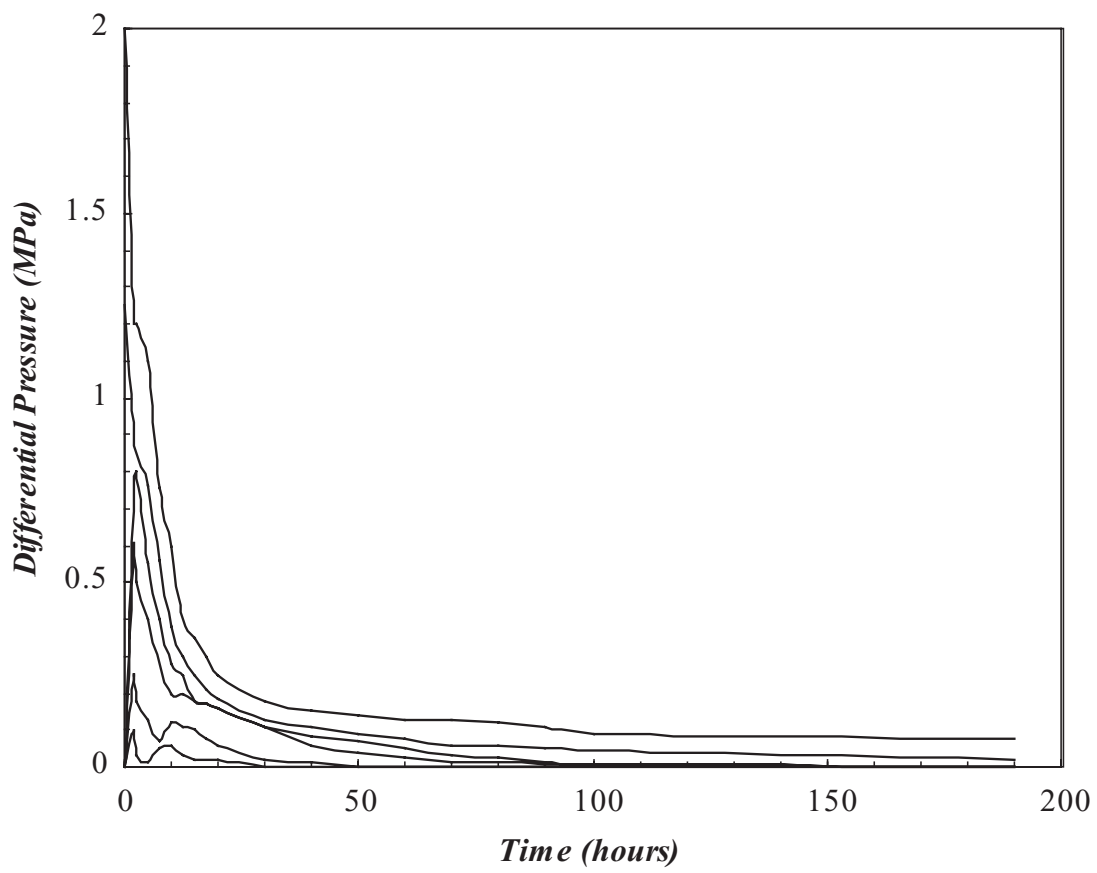

Figure 6.5. Experimental data for unsteady blowdown problem by Maini and Sarma 1994. The pressure drop across 6 segments is presented with the greatest pressure drop across the segment at the outlet (open end) and smallest drops across the segment at the closed end.

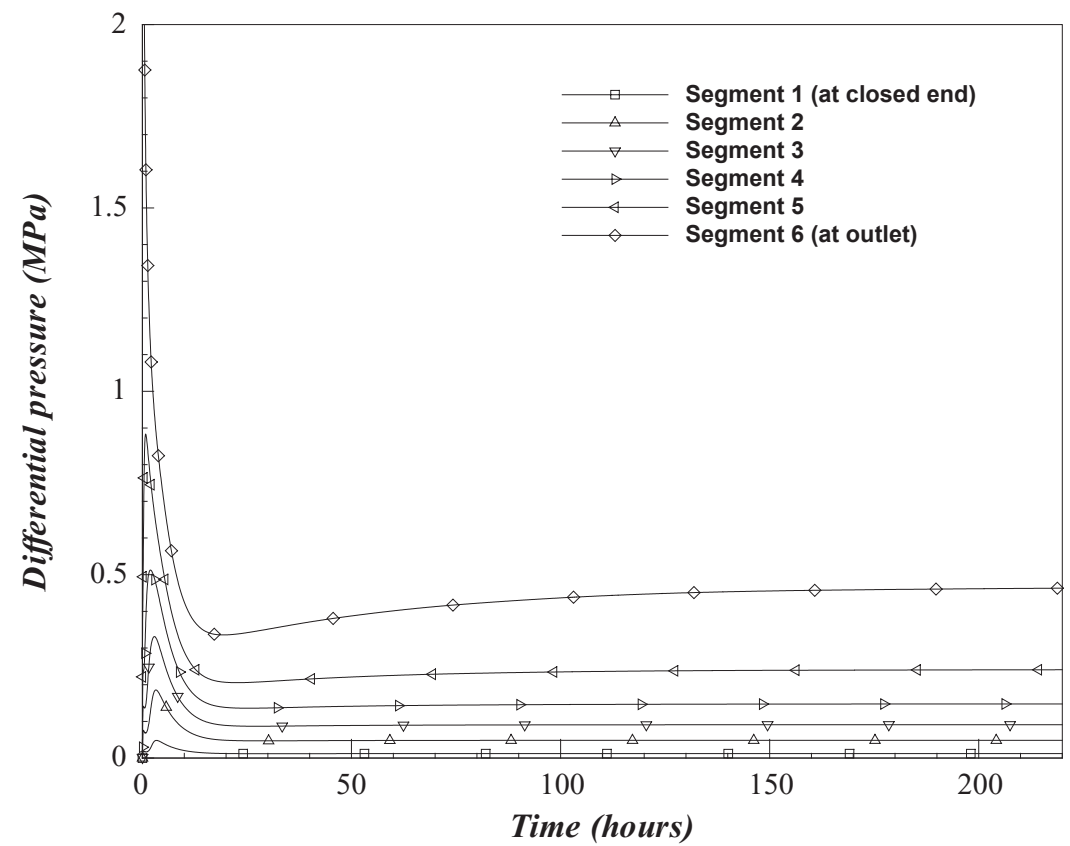

Figure 6.6. Numerical result for the differential pressure for nonlinear relaxation $J_{1}$ and $J_{2}$ $\left(J_{1}=200 * \exp (-500 * \phi)+5, J_{2}=-1.99 * \exp (-200 * \phi)-0.01\right)$.

The blowdown experiment of Maini and Sarma (1994) is discussed and prepared for comparison with theory in section 9 of Part I. A graph of the experiment is shown in figure 6.1 of Part I. There are 7 pressure taps along the length of the sand pack; this corresponds to 6 segments between taps, as shown in figure 6.7. 


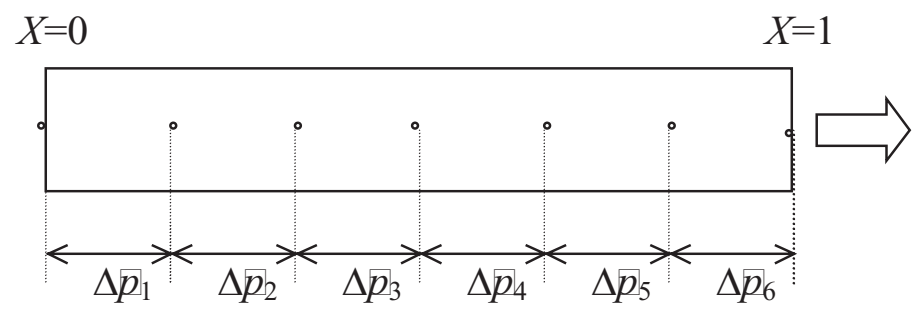

Figure 6.7. Schematic design of the seven pressure taps and six segments between the closed end $(X=0)$ and the open end $(X=1)$ at which production occurs.

The evolution of the differential pressure across each segment was recorded and is shown in figure 6.5. The pressure at the outlet $x=L$ in this experiment was dropped suddenly from

$$
p=4.83 \mathrm{MPa} \text { to } p_{L}=0.75 \mathrm{MPa} \text {. }
$$

The theoretical result for the nonlinear relaxation theory is given in figure 6.6. A somewhat better than qualitative agreement is evident. The predicted differential pressure across each segment is higher at long times than in the experiments, as they were in the depletion experiments shown in figure 6.1. Our tentative explanation is that the lower differential pressure observed in the experiments is due to coalescence and percolation of free gas not allowed in the theory.

The pressure decay for the constant relaxation time theory shown in figure 6.8 is much slower than in the experiments.

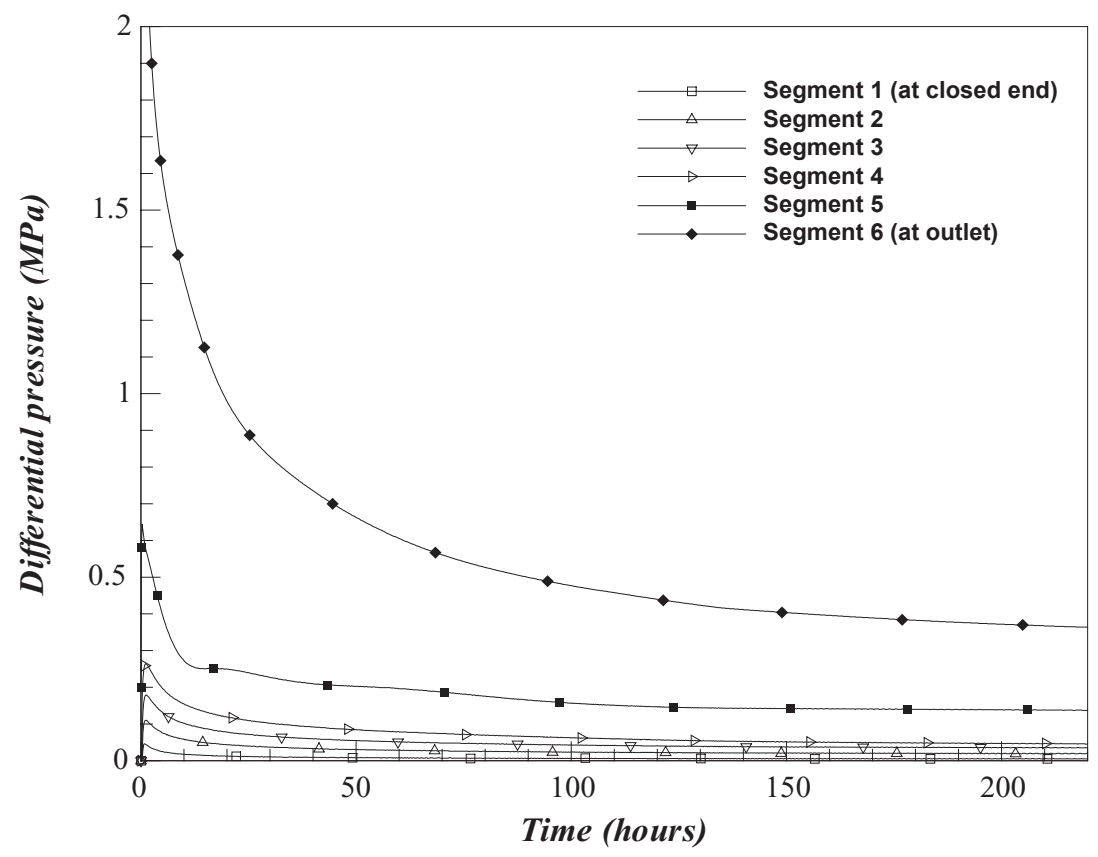

Figure 6.8. Numerical result for the differential pressure at constant relaxation $J_{1}$ and $J_{2}\left(J_{1}=200\right.$, $\left.J_{2}=-18\right)$.

The evolution of the gas fraction at early times predicted by the nonlinear and constant relaxation time theories are in figure 6.9 and 6.10, respectively. The evolution of gas at early times is markedly suppressed by the nonlinear theory modeling nucleation. Gas fractions were not measured in the experiments. 


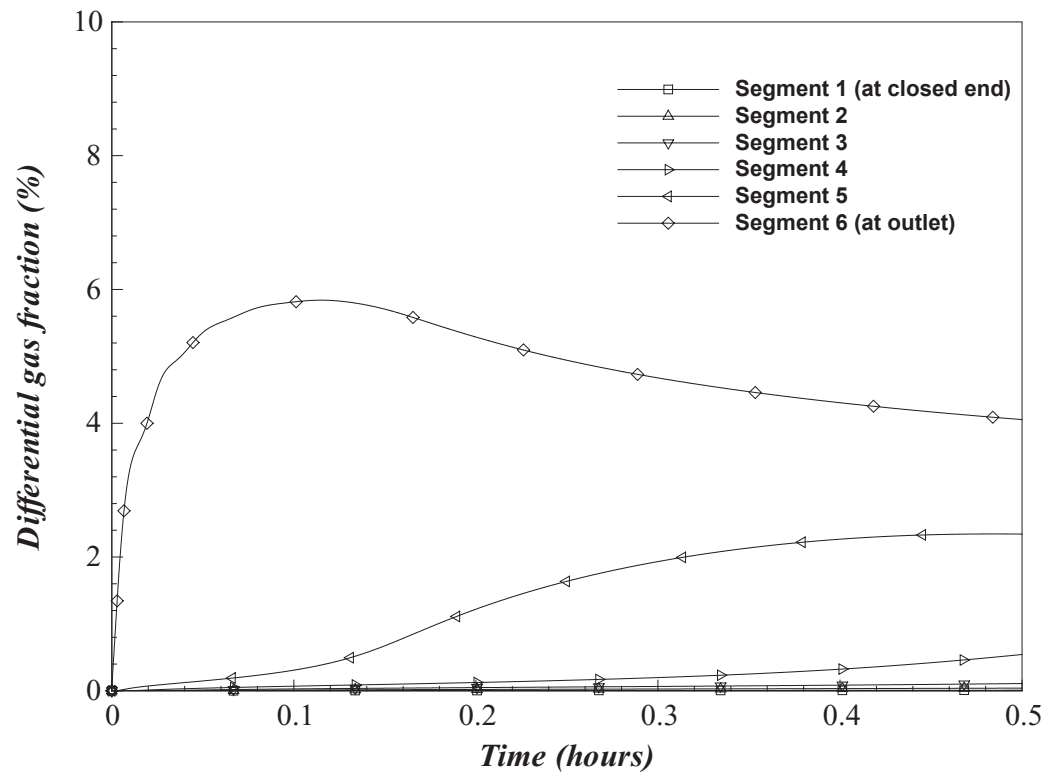

Figure 6.9. The differential gas fraction at 6 segments for short time $\left(J_{1}=200 * \exp (-500 * \phi)+5, J_{2}=\right.$ $-1.99 * \exp (-200 * \phi)-0.01)$.

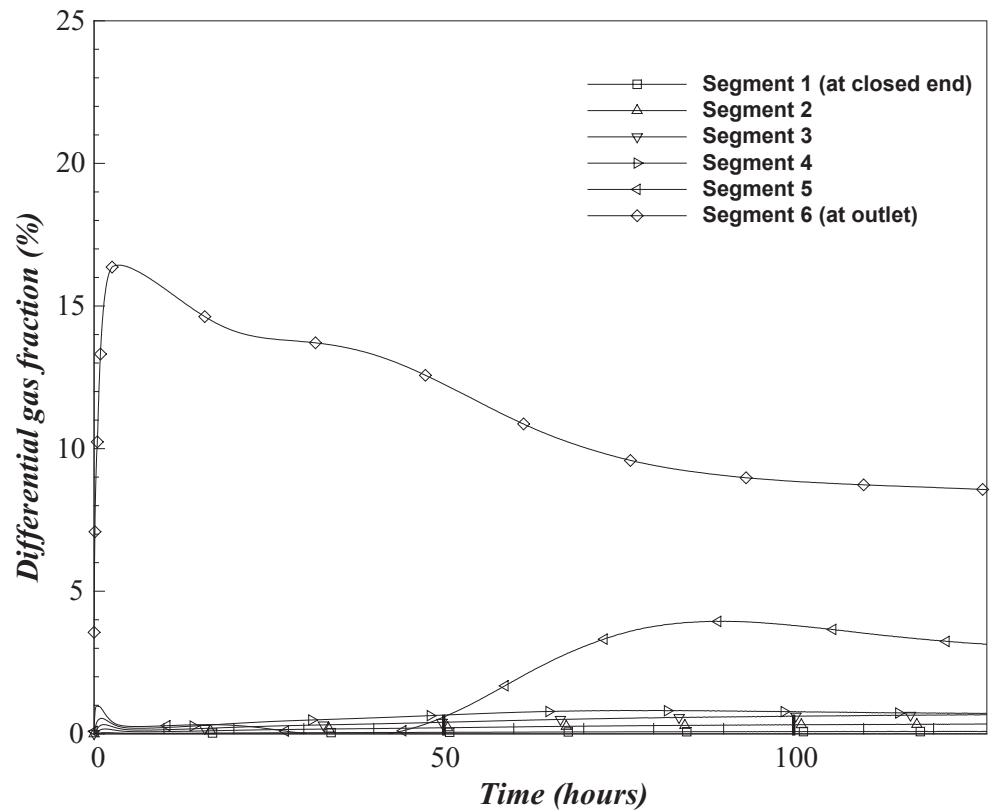

Figure 6.10. Numerical result for the differential gas fraction at constant relaxation $J_{1}$ and $J_{2}\left(J_{1}=200\right.$, $\left.J_{2}=-18\right)$.

The cumulative oil production in the blowdown was measured and is shown in figure 6.1. The cumulative production may be obtained as the integral over time of the rate of production

$$
\rho_{0} \stackrel{\circ}{Q}(t)=\rho_{0} \int_{0}^{t} Q(t) \mathrm{d} t, \quad \stackrel{\circ}{Q}=\frac{A \lambda(1-\phi) d P}{d X}
$$

at the open end of the sand pack (see section 9, Part I). Theoretical results for oil production are also presented in figure 6.11. The nonlinear relaxation time theory represents very well the production at early times. The discrepancy at long times is again compatible with the idea that oil 
production in the experiments is suppressed by the percolation of free gas not allowed in the theories.

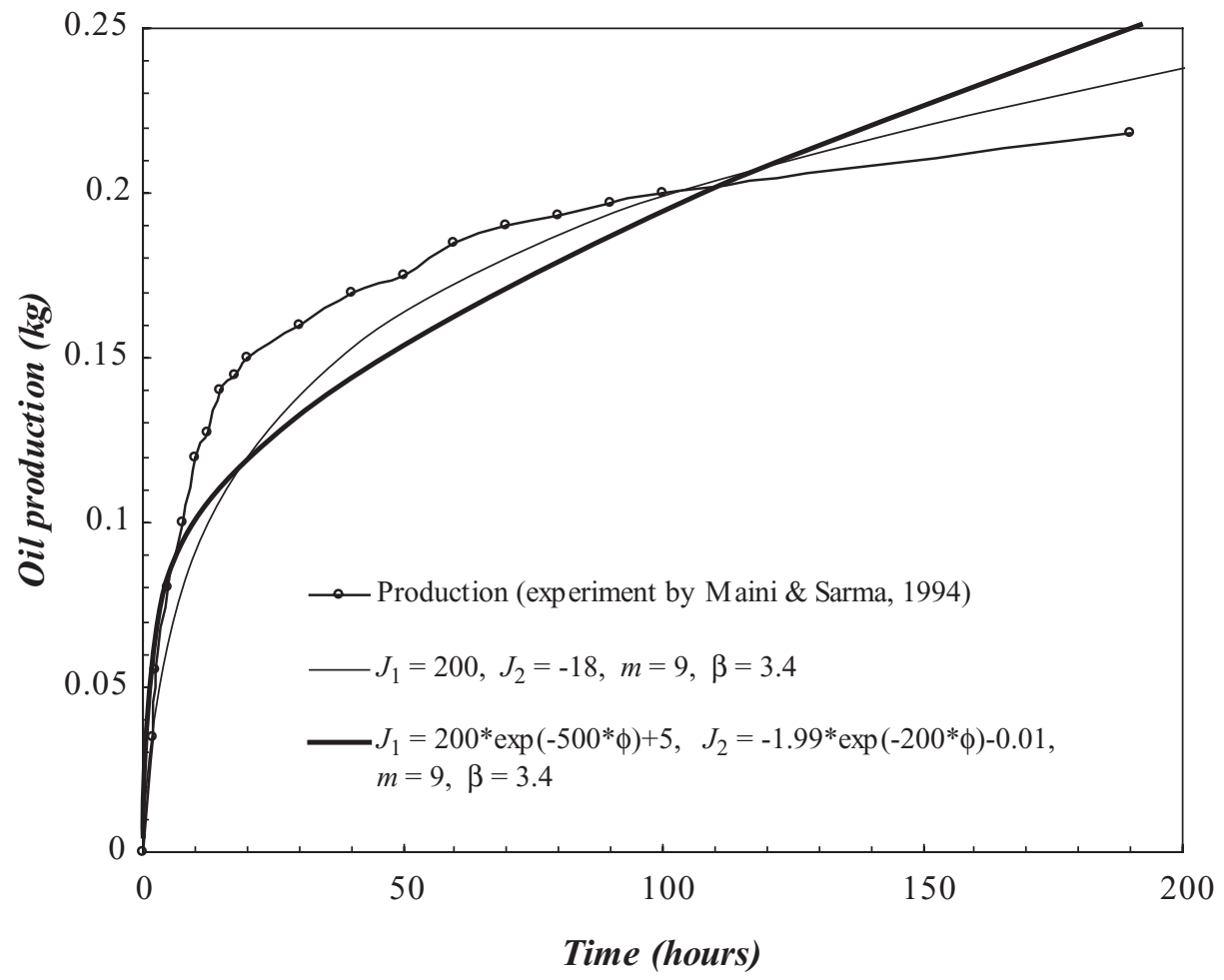

Figure 6.11. Oil production $\rho_{0} \mathrm{Q}(\mathrm{t})$ vs. time. Comparison of numerical results for nonlinear relaxation and constant relaxation with experimental data by Maini and Sarma (1994) in the blowdown experiment.

\section{Conclusions}

A summary of the main points is in the abstract of this paper. We stand by the conclusions already presented in Part I with the caveat that the introduction of the continuum theory of nucleation developed here represents an advance in the evolution of this theory. Future work points to the incorporation of the compressibility of live oil, and an explicit recognition of the effects of liberation of dissolved gases on the oil and transfer properties neglected here. The most important feature lacking in the present work is the coalescence of dispersed gas and the percolation of free gas streams. It is not yet clear how many of these features can be incorporated in a simple empirically based and mathematically elegant description of the flow of foamy oil in porous media.

\section{Acknowledgement}

The work of D.D. Joseph, T. Ko and R. Bai was supported in part by the DOE (Engineering Research Program of the Dept of Basic Energy Sciences), the NSF grants from Chemical Transport Systems and GOALI, and the Minnesota Supercomputer Institute. The work of A.M. Kamp was supported by PDVSA Intevep S.A.. 


\title{
Appendix A: \\ Numerical method for the solution of (3.3), (3.4) and (3.5) for sand pack problems
}

\author{
by T. Ko.
}

To solve the fully implicit constitutive equations, we write again (3.3), (3.4) and (3.5) as follows:

$$
\begin{gathered}
U^{\prime}=\left\{\left(\frac{1}{1+\Psi^{n-1}}\right)^{m} /\left(P^{n-1}+1\right)^{2}\right\} \frac{\partial P^{n-1}}{\partial X} \\
\frac{1}{1+\Psi}\left[\frac{\Psi^{n}-\Psi^{n-1}}{\Delta T}+\frac{1}{2} U^{\prime}\left\{\frac{\partial \Psi^{n}}{\partial X}+\frac{\partial \Psi^{n-1}}{\partial X}\right\}\right]=\frac{\partial U^{\prime}}{\partial X} \\
U^{\prime \prime}=\left\{\left(\frac{1}{1+\Psi^{n}}\right)^{m} /\left(P^{n-1}+1\right)^{2}\right\} \frac{\partial P^{n-1}}{\partial X} \\
J_{2} \frac{P^{n}-P^{n-1}}{\Delta T}+\frac{1}{2} J_{2} \mathrm{U}^{n}\left\{\frac{\partial P^{n}}{\partial X}+\frac{\partial P^{n-1}}{\partial X}\right\}-\frac{1}{2}\left(P^{n}+P^{n-1}\right)=-J_{1}\left\{\frac{\Psi^{n}-\Psi^{n-1}}{\Delta T}+U^{n} \frac{\partial \Psi^{n}}{\partial X}\right\}-\beta \Psi^{n}
\end{gathered}
$$

where the superscript $n$ indicates the time level, and $U^{\prime}$ is intermediate velocity. Three variables, $U, P$ and $\Psi$, are decoupled in the rewritten four equations. The $\Psi$ in (A.2) and $P$ in (A.4) are integrated using the Crank-Nicolson method (second-order accuracy). Before solving (A.2), the velocity is updated with the values of the pressure and gas fraction at a previous time step through (A.1). The velocity is calculated again in (A.3) with the new value of $\Psi$ obtained from (A.2) and used in (A.4) to calculate $P$ with the updated value of $\Psi$. By calculating (3.3) two times, we can get converged values for each time step more quickly.

The constitutive equations are discretized using a Galerkin method. The weak formulations of the constitutive equations are derived by multiplying them by a corresponding weighting function and integrating over the spatial domain of a problem. The one-dimensional uniform grid is used in a calculation domain. In (A.2) and (A.4), the global matrix of the equations has a Tridiagonal matrix formation.

The main iteration is the loop of calculation for the four-step equations (A.1), (A.2), (A.3) and (A.4) to get the converged value of variables $U, P$ and $\Psi$ at each time step. The subiteration is done to linearize the non-linear term for $\Psi$ in equation (A.2). The solution at steady state is sought through time marching of the corresponding unsteady governing equation. Therefore, the value of $1 / \Delta t$ can be considered as an inertial relaxation factor of steady SIMPLE algorithm Patankar (1980). 


\section{References}

D. Joseph, A. Kamp and R.Bai, 2002. Modeling foamy oil flow in porous media, Int. J. Multiphase Flow 28, pp.1659-1686.

Kumar, Pooladi-Darvish and Okazawa, 2000. An investigation into enhanced recovery under solution gas drive in heavy oil reservoirs, SPE 59336, for SPE/DOE Improved Oil Recovery Symposium in Tulsa, Oklahoma, 3-5 April 2000.

Maini B.B.\& Sarma, H, 1994. Role of Nonpolar Foams in Production of Heavy Oils, Advances in Chemistry Series, 242, 405-420.

Patankar, S.V., 1980. Numerical Heat Transfer and Fluid Flow. Hemisphere Publishing Corporation, 67-68. 